\title{
Deferred Statistical Cluster Points of Real Valued Sequences
}

\author{
Müjde Yılmaztürk, Özgür Mızrak*, Mehmet Küçükaslan \\ Department of Mathematics, Arts and Science Faculty, Mersin University, Mersin, 33343, Turkey \\ *Corresponding Author: mizrak@mersin.edu.tr
}

Copyright (C)2013 Horizon Research Publishing All rights reserved.

\begin{abstract}
In this paper, the concept of deferred statistical cluster points of real valued sequences is defined and studied by using deferred density of the subset of natural numbers. For $p(n)$ and $q(n)$ satisfying certain conditions, we give some results for the set of deferred statistical cluster points $\Gamma_{D_{p, q}}(x)$. We provide some counter examples regarding $\Gamma_{D_{p, q}}(x)$. Also we obtain some inclusion results for $\Gamma_{D_{p, q}}(x)$. At last we consider the case $q(n)=\lambda(n)$ and $p(n)=\lambda(n-1)$ where the sequence $\lambda=\{\lambda(n)\}$ is strictly increasing sequence of positive natural numbers with $\lambda(0)=0$.
\end{abstract}

Keywords Natural density, statistical cluster points, statistically convergent sequence

\section{Introduction and notations}

The concept of statistical convergence was introduced by H. Fast [8] and I.J. Steinhaus [23] independently in 1951. Since then, this subject was applied in different areas of mathematics such as in number theory by P. Erdös-G. Tenenbaum [7] and summability theory by A.R. Freedman-J.J. Sember-M. Raphael [9], etc.

Some properties of statistical convergence were studied by J. Conner in [3, 4], J.A. Fridy [10], J.A. FridyC. Orhan [12], T. Salat [21], I.J. Schenberg [22] and the others.

This subject is closely related to the subject of asymptotic (natural) density of the subset of natural numbers [2] and its root goes to A. Zygmund [25].

By using asimptotic density, the concept of statistical cluster points of real valued sequences was first introduced by J.A. Fridy [11]. Some generalizations of this concept have been studied by using regular summability methods in [5, 6, 13, 16, 20, 24].

In 1932, R.P. Agnew [1] defined the deferred Cesaro mean $D_{p, q}$ of the sequence $x=\left(x_{k}\right)$ by

$$
\left(D_{p, q} x\right)_{n}:=\frac{1}{q(n)-p(n)} \sum_{p(n)+1}^{q(n)} x_{k}
$$

where $\{p(n)\}$ and $\{q(n)\}$ are sequences of positive nat- ural numbers satisfying

$$
p(n)<q(n) \text { and } \lim _{n \rightarrow \infty} q(n)=\infty
$$

Let $K$ be a subset of $\mathbb{N}$, and denote the set $\{k: p(n)<$ $k \leq q(n), k \in K\}$ by $K_{p, q}(n)$. The deferred density of $K$ is defined by

$$
\delta_{p, q}(K):=\lim _{n \rightarrow \infty} \frac{1}{q(n)-p(n)}\left|K_{p, q}(n)\right|
$$

whenever the limit exists. The vertical bars in (2) indicate the cardinality of the set $K_{p, q}(n)$.

Because of $\delta_{p, q}(K)$ does not exists for all $K \subset \mathbb{N}$, it is convenient to use upper deferred asymptotic density of $K$, defining by

$$
\delta_{p, q}^{*}(K)=\limsup _{n \rightarrow \infty} \frac{|\{k: p(n)+1 \leq k \leq q(n), k \in K\}|}{q(n)-p(n)} .
$$

It is clear that,

i) if $\delta_{p, q}(K)$ exists, then $\delta_{p, q}(K)=\delta_{p, q}^{*}(K)$,

ii) $\delta_{p, q}(K) \neq 0$ if and only if $\delta_{p, q}^{*}(K)>0$,

iii) if $K \subset M$, then $\delta_{p, q}^{*}(K) \leq \delta_{p, q}^{*}(M)$

A real valued sequence $x=\left(x_{n}\right)$ is deferred statistical convergent to $l$, if the limit

$$
\lim _{n \rightarrow \infty} \frac{1}{q(n)-p(n)}\left|\left\{p(n)<k \leq q(n):\left|x_{k}-l\right| \geq \varepsilon\right\}\right|=0,
$$

exists for every $\varepsilon>0$. (see $[14,15])$.

It is clear that:

iv) If $q(n)=n, p(n)=0$ then (2) and (3) coincide the usual asymptotic density and statistical convergence respectively [10].

v) If $q(n)=\lambda(n), p(n)=n-\lambda(n)$ for the sequence $\lambda(n)$ satisfying $\lambda(n+1) \leq \lambda(n)+1$ and $\lambda(1)=1$, then (2) and (3) coincide $S_{\lambda}$-density and $S_{\lambda}$-convergence which was defined and studied by M.Mursaleen [17]. 
vi) If $q(n)=\lambda(n), p(n)=0$ for the sequence $\lambda(n)$ such that it is strictly increasing sequence of natural numbers with $\lambda(0)=0$, then (2) and (3) coincide $S C_{\lambda}$-density and $S C_{\lambda}$-convergence which was defined by [18].

Definition 1.1. The number $\gamma$ is called deferred statistical cluster point of $x=\left(x_{k}\right)$, for every $p(n)$ ad $q(n)$ satisfying (1), if for every $\varepsilon>0$ the set

$$
\left\{p(n)<k \leq q(n):\left|x_{k}-\gamma\right|<\varepsilon\right\}
$$

does not have deferred density zero i.e.,

$$
\lim _{n \rightarrow \infty} \frac{\left|\left\{p(n)<k \leq q(n):\left|x_{k}-\gamma\right|<\varepsilon\right\}\right|}{q(n)-p(n)} \neq 0
$$

and the set of deferred statistical cluster points of the sequence $x=\left(x_{k}\right)$ is denoted by $\Gamma_{D_{p, q}}(x)$, i.e.,

$$
\Gamma_{D_{p, q}}(x):=\{\gamma: \gamma \text { satisfies }(4)\}
$$

This definition is generalized version of the statistical cluster point definition given by J.A. Fridy in [11].

\section{Main Results}

\subsection{Some properties of deferred statistical cluster points}

In this section, some topological properties of deferred statistical cluster points of the real valued sequences are giong to be investigated.

Theorem 2.1. If the sequence $x=\left(x_{n}\right)$ is deferred statistical convergence to $l$, then $\Gamma_{D_{p, q}}(x)$ contains only the elements $l$.

Proof. Assume that the sequence $x=\left(x_{n}\right)$ is deferred statistical convergence to $l$. Then, for every $\varepsilon>0$, the limit relation

$$
\lim _{n \rightarrow \infty} \frac{\left|\left\{k: p(n)+1 \leq k \leq q(n), \quad\left|x_{k}-l\right| \geq \varepsilon\right\}\right|}{q(n)-p(n)}=0
$$

hold. It means that

$$
\lim _{n \rightarrow \infty} \frac{\left|\left\{k: p(n)+1 \leq k \leq q(n), \quad\left|x_{k}-l\right|<\varepsilon\right\}\right|}{q(n)-p(n)}=1 \neq 0 .
$$

Therefore, $l \in \Gamma_{D_{p, q}}(x)$. Now let us assume that the set $\Gamma_{D_{p, q}}(x)$ contains $l^{\prime}$ which different from $l$, i.e., $l \neq$ $l^{\prime}$. Take into consider $\varepsilon=\frac{1}{2}\left|l-l^{\prime}\right|$. Since $x=\left(x_{n}\right)$ is deferred statistical convergence to $l$, then (5) is hold for this $\varepsilon$. It means that deferred asymptotic density of the elements $x=\left(x_{n}\right)$ belonging to the $\varepsilon$-neigborhood of $l$ is 1. Consequently, the deferred asymptotic density of the elements of $\left(x_{n}\right)$ belonging to the $\varepsilon$-neighborhood of $l^{\prime}$ is zero. That is,

$$
\lim _{n \rightarrow \infty} \frac{\left|\left\{k: p(n)+1 \leq k \leq q(n), \quad\left|x_{k}-l^{\prime}\right|<\varepsilon\right\}\right|}{q(n)-p(n)}=0 .
$$

This is contradiction to assumption on $l^{\prime}$.

Remark 2.1. The inverse of Theorem 2.1 is not true.
There exists a sequence such that the set of deferred statistical cluster points has unique elements but it is not deferred statistical convergence to this point. Let us consider the sequence $x=\left(x_{n}\right)$ where

$$
x_{n}:= \begin{cases}\frac{1}{n}, & n \text { even } \\ n, & n \text { odd }\end{cases}
$$

It is clear that $0 \in \Gamma_{D_{0, n}}(x)$ but it is not deferred statistical convergence to zero.

Remark 2.2. Assume that the sequence $x=\left(x_{n}\right)$ is monotone increasing (decreasing). If $\sup x_{n}<\infty$ $\left(\inf x_{n}<\infty\right)$, then $\sup x_{n} \in \Gamma_{D_{p, q}}(x), \quad\left(\inf x_{n} \in\right.$ $\left.\Gamma_{D_{p, q}}(x)\right)$ respectively.

Proof. Here we will give the proof for only the monotone increasing sequence. From the definition of supremum for any $\varepsilon>0$ there exists a $n_{0} \in \mathbb{N}$ such that the following inequality

$$
\sup x_{n}-\varepsilon<x_{n_{0}} \leq \sup x_{n}
$$

hold.

Since the sequence is monotone increasing, then we have

$$
\sup x_{n}-\varepsilon<x_{n_{0}}<x_{n} \leq \sup x_{n}<\sup x_{n}+\varepsilon
$$

for all $n>n_{0}$. It means that for any $\varepsilon>0$ there exist a $n_{0}(\varepsilon) \in \mathbb{N}$ such that the inequality

$$
\left|x_{n}-\sup x_{n}\right|<\varepsilon
$$

holds for all $n>n_{0}$. From this discussion the following inclusion

$$
\mathbb{N}-\left\{1,2, \ldots, n_{0}\right\} \subset\left\{n:\left|x_{n}-\sup x_{n}\right|<\varepsilon\right\}
$$

holds. Since $\delta_{p, q}\left(\mathbb{N}-\left\{1,2, \ldots, n_{0}\right\}\right)=1$, then $\delta_{p, q}\left(\left\{n:\left|x_{n}-\sup x_{n}\right|<\varepsilon\right\}\right) \neq 0$. This gives the desired proof.

Recall that the distance between $A \subset \mathbb{R}$ and $B \subset \mathbb{R}$ is

$$
d(A, B)=\inf \{|a-b|: a \in A, b \in B\}
$$

Theorem 2.2. Let $x=\left(x_{n}\right)$ be a real valued sequence. If $\Gamma_{D_{p, q}}(x) \neq \varnothing$, then $d\left(\Gamma_{D_{p, q}}(x), x\right)=0$.

Proof. Assume that $\Gamma_{D_{p, q}}(x) \neq \varnothing$. Let us consider an arbitrary element $y \in \Gamma_{D_{p, q}}(x)$. Then, we have for an arbitrary positive $\varepsilon$,

$$
\lim _{n \rightarrow \infty} \frac{\left|\left\{k: p(n)+1 \leq k \leq q(n), \quad\left|x_{k}-y\right|<\varepsilon\right\}\right|}{q(n)-p(n)} \neq 0 .
$$

So, the set $A_{\varepsilon}:=\left\{x_{k}:\left|x_{k}-y\right|<\varepsilon\right\}$ has at least countable elements of $x=\left(x_{n}\right)$ for an arbitrary positive $\varepsilon$. Therefore,

$$
0 \leq d\left(\Gamma_{D_{p, q}}(x), x\right)=\inf \left\{\left|y-x_{k}\right|: k \in \mathbb{N}\right\} \leq \varepsilon
$$

is hold. This gives the desired proof.

Theorem 2.3. If $\Gamma_{D_{p, q}}(x) \neq \varnothing$ for any $p(n)$ and $q(n)$, then $\Gamma_{D_{p, q}}(x)$ is closed. 
Proof. Let us assume that $\Gamma_{D_{p, q}}(x) \neq \varnothing$ for any $p(n)$ and $q(n)$. It is enough to show that $\mathbb{R} \backslash \Gamma_{D_{p, q}}(x)$ is an open set. Let $y \in \mathbb{R} \backslash \Gamma_{D_{p, q}}(x)$ be an arbitrary point. Since $y \notin \Gamma_{D_{p, q}}(x)$, then there exists an $\varepsilon>0$ such that

$$
\delta_{p, q}\left(\left\{p(n)<k \leq q(n):\left|x_{k}-y\right|<\varepsilon\right\}\right)=0 .
$$

If we denote the open interval $(y-\varepsilon, y+\varepsilon)$ by $A$, then we have

$$
\delta_{p, q}\left(\left\{p(n)<k \leq q(n): x_{k} \in A\right\}\right)=0 .
$$

If we choose $\varepsilon_{y}:=\frac{1}{2} \inf \left\{\left|x_{k}-y\right|: x_{k} \in A\right\}$, then it is clear that $\varepsilon_{y}<\varepsilon$ and $\left(y-\varepsilon_{y}, y+\varepsilon_{y}\right) \subset \mathbb{R} \backslash \Gamma_{D_{p, q}}(x)$. It means that $y$ is an arbitrary interior point of $\mathbb{R} \backslash \Gamma_{D_{p, q}}(x)$. Therefore $\mathbb{R} \backslash \Gamma_{D_{p, q}}(x)$ is an open set.

Theorem 2.4. Let $x=\left(x_{n}\right)$ be a real valued sequence and $\gamma \in \mathbb{R}$ be an arbitrary fixed point. If $d(\gamma, x) \neq 0$, then $\gamma \notin \Gamma_{D_{p, q}}(x)$ for any $p(n)$ and $q(n)$.

Proof. From the hypthesis we have

$$
d(\gamma, x):=\inf \left\{\left|x_{k}-\gamma\right|: k \in \mathbb{N}\right\}=m>0 .
$$

From this assumption the inequality

$$
\left|x_{k}-\gamma\right| \geq m
$$

hold for all $k \in \mathbb{N}$.

It means that the open interval $(\gamma-m, \gamma+m)$ has no elements of the sequence $x=\left(x_{n}\right)$. So, we have

$$
\delta_{p, q}\left(\left\{p(n)<k \leq q(n): x_{k} \in(\gamma-m, \gamma+m)\right\}\right)=0
$$

therefore, if we choose an arbitrary $\varepsilon<m$ then the relation

$$
\delta_{p, q}\left(\left\{p(n)<k \leq q(n):\left|x_{k}-\gamma\right|<\varepsilon\right\}\right)=0
$$

hold. Otherwise it contradicts with (6) since the inclusion

$$
\begin{aligned}
\{p(n)<k \leq & \left.q(n):\left|x_{k}-\gamma\right|<\varepsilon\right\} \subset \\
& \subset\left\{p(n)<k \leq q(n):\left|x_{k}-\gamma\right|<m\right\} .
\end{aligned}
$$

Remark 2.3. If $d(\gamma, x)=0$, it is not necessarily $\gamma \in$ $\Gamma_{p, q}(x)$.

Let us consider the sequence $x=\left(x_{n}\right)=\left(\frac{1}{n}\right)$ for all $n \in \mathbb{N}$. If we take $\gamma=\frac{1}{2}$, then $d\left(\frac{1}{2}, \frac{1}{n}\right)=0$ but $\frac{1}{2} \notin$ $\Gamma_{D_{p, q}}(x)=\{0\}$ when $q(n)=n$ and $p(n)=0$.

Theorem 2.5. Let $x=\left(x_{n}\right)$ be a real valued sequence and $A \subset \mathbb{R}$ be an arbitrary set. If $d(A, x) \neq 0$, then

$$
A \cap \Gamma_{D_{p, q}}(x)=\varnothing .
$$

Proof. If the subset $A \subset \mathbb{R}$ is a singleton, then the proof is obtained from Theorem 2.4. Let $a^{*} \in A$ be an arbitrary element. There is $m>0$ such that

$$
\left|a^{*}-x_{k}\right|>m
$$

since $d(A, x)>0$. So, the intervals $\left(a^{*}-m, a^{*}+m\right)$ has no elements of $x=\left(x_{n}\right)$. Therefore, if we choose $\varepsilon<$ $m$, the set $\left(a^{*}-m, a^{*}+m\right)$ contains no element of the sequence. Consequently, we have

$$
\delta_{p, q}\left(\left\{p(n)<k \leq q(n):\left|a^{*}-x_{k}\right|<\varepsilon\right\}\right)=0
$$

and $a^{*} \notin \Gamma_{D_{p, q}}(x)$.
Remark 2.4. If $d(A, x)=0$, it is not necessarily $A \cap$ $\Gamma_{D_{p, q}}(x) \neq \varnothing$

Let us consider the sequence $x=\left(x_{n}\right)=\left(\frac{1}{n}\right)$ for all $n \in \mathbb{N}$ and $A=(0, \infty)$. It clear that $A \cap \Gamma_{D_{p, q}}(x)=\varnothing$ but $d(A, x)=0$.

\subsection{Some inclusion results for $\Gamma_{D_{p, q}}(x)$}

Thorought this section, we consider the sequences of positive natural numbers $p(n), p^{\prime}(n), q(n)$ and $q^{\prime}(n)$

Denote the sets for only simplicitly $E$ := $\{p(n): n \in \mathbb{N}\}, \quad E^{\prime} \quad:=\quad\left\{p^{\prime}(n): n \in \mathbb{N}\right\}, \quad F \quad:=$ $\{q(n): n \in \mathbb{N}\}$ and $F^{\prime}:=\left\{q^{\prime}(n): n \in \mathbb{N}\right\}$.

Theorem 2.6. If the set $F^{\prime} \backslash F$ is finite and

$$
\lim _{n \rightarrow \infty} \frac{q(n)-p(n)}{q^{\prime}(n)-p(n)}=d \neq 0
$$

hold. Then, $\delta_{p, q^{\prime}}(K) \neq 0$ implies $\delta_{p, q}(K) \neq 0$ for every $K \subseteq \mathbb{N}$.

Proof. Since the set $F^{\prime} \backslash F$ is finite, then there exists a positive natural number $N$ such that

$$
\left\{q^{\prime}(n): n \geq N\right\} \subset\{q(n): n \in \mathbb{N}\} .
$$

For $n \geq N$ let $j(n)$ be a strictly increasing sequence such that $q^{\prime}(n):=q(j(n))$. If $\delta_{p, q^{\prime}}(K) \neq 0$ then the relation

$\delta_{p, q^{\prime}}^{*}(K)=\limsup _{n \rightarrow \infty} \frac{\left|\left\{p(n)+1 \leq k \leq q^{\prime}(n): k \in K\right\}\right|}{q^{\prime}(n)-p(n)}>0$

holds. So, we have

$$
\begin{aligned}
& \frac{|\{p(n)+1 \leq k \leq q(j(n)): k \in K\}|}{q(j(n))-p(n)} \leq \\
& \leq \frac{q(n)-p(n)}{q(j(n))-p(n)} \cdot \frac{|\{p(n)+1 \leq k \leq q(n): k \in K\}|}{q(n)-p(n)}
\end{aligned}
$$

and

$\delta_{p, q}^{*}(K)=\limsup \frac{|\{p(n)+1 \leq k \leq q(n): k \in K\}|}{q(n)-p(n)}>0$.

Hence $\delta_{p, q}(K) \neq 0$ and the proof is obtained.

Corollary 2.1. Let us assume that

$$
\lim _{n \rightarrow \infty} \frac{q(n)-p(n)}{q^{\prime}(n)-p(n)}=d \neq 0
$$

hold. Then, the followings are true:

i) If $F^{\prime} \backslash F$ is finite, then

$$
\Gamma_{D_{p, q}}(x) \supset \Gamma_{D_{p, q^{\prime}}}(x),
$$

ii) If $F^{\prime} \triangle F$ is finite, then

$$
\Gamma_{D_{p, q}}(x)=\Gamma_{D_{p, q^{\prime}}}(x)
$$

Theorem 2.7. If $E^{\prime} \backslash E$ is finite and

$$
\lim _{n \rightarrow \infty} \frac{q(n)-p^{\prime}(n)}{q(n)-p(n)}=d \neq 0
$$

hold. Then, $\delta_{D_{p, q}}(K) \neq 0$ implies $\delta_{D_{p^{\prime}, q}}(K) \neq 0$ for every $K \subseteq \mathbb{N}$. 
Proof. If $E^{\prime} \backslash E$ is finite, then there exists a positive natural number $N$ such that

$$
\left\{p^{\prime}(n): n \geq N\right\} \subset\{p(n): n \in \mathbb{N}\}
$$

hold.

For $n \geq N$ let $j(n)$ be monotone increasing such that $p^{\prime}(n)=p(j(n))$. If $\delta_{D_{p, q}}(K) \neq 0$ then

$\delta_{D_{p, q}}^{*}(K)=\limsup _{n \rightarrow \infty} \frac{|\{p(n)+1 \leq k \leq q(n): k \in K\}|}{q(n)-p(n)}>0$.

From this we have

$$
\begin{aligned}
& \frac{|\{p(n)+1 \leq k \leq q(n): k \in K\}|}{q(n)-p(n)} \leq \\
\leq & \frac{q(n)-p(j(n))}{q(n)-p(n)} \cdot \frac{|\{p(j(n))+1 \leq k \leq q(n): k \in K\}|}{q(n)-p(j(n))}
\end{aligned}
$$

and

$\delta_{D_{p, q}}^{*}(K)=\limsup \frac{\left|\left\{p^{\prime}(n)+1 \leq k \leq q(n): k \in K\right\}\right|}{q(n)-p^{\prime}(n)}>0$.

It gives $\delta_{D_{p^{\prime}, q}}(K) \neq 0$ and we obtained desired result.

Corollary 2.2. Under the assumption of Theorem 2.7 the following statements are true:

i) If $E^{\prime} \backslash E$ is finite, then

$$
\Gamma_{D_{p^{\prime}, q}}(x) \supset \Gamma_{D_{p, q}}(x),
$$

ii) If $E^{\prime} \triangle E$ is finite, then

$$
\Gamma_{D_{p^{\prime}, q}}(x)=\Gamma_{D_{p, q}}(x) .
$$

Theorem 2.8. Let us assume that

$$
p(n) \leq p^{\prime}(n)<q^{\prime}(n) \leq q(n)
$$

and

$$
\lim _{n \rightarrow \infty} \frac{q(n)-p(n)}{q^{\prime}(n)-p^{\prime}(n)}=d \neq 0
$$

hold. Then, $\delta_{D_{p^{\prime}, q^{\prime}}}(K) \neq 0$ implies $\delta_{D_{p, q}}(K) \neq 0$ for every $K \subseteq \mathbb{N}$.

Proof. If $\delta_{D_{p^{\prime}, q^{\prime}}}(K) \neq 0$, then we have

$$
\begin{aligned}
& \delta_{D_{p^{\prime}, q^{\prime}}}^{*}(K)= \\
& \quad=\limsup _{n \rightarrow \infty} \frac{\left|\left\{p^{\prime}(n)+1 \leq k \leq q^{\prime}(n): k \in K\right\}\right|}{q^{\prime}(n)-p^{\prime}(n)}>0,
\end{aligned}
$$

and the relation

$$
\begin{gathered}
\frac{\left|\left\{p^{\prime}(n)+1 \leq k \leq q^{\prime}(n): k \in K\right\}\right|}{q^{\prime}(n)-p^{\prime}(n)} \leq \\
\leq \frac{q(n)-p(n)}{q^{\prime}(n)-p^{\prime}(n)} \cdot \frac{|\{p(n)+1 \leq k \leq q(n): k \in K\}|}{q(n)-p(n)}
\end{gathered}
$$

hold. Therefore,

$\delta_{D_{p, q}}^{*}(K)=\limsup \frac{|\{p(n)+1 \leq k \leq q(n): k \in K\}|}{q(n)-p(n)}>0$.

Hence $\delta_{D_{p, q}}(K) \neq 0$, and the proof is ended.
Corollary 2.3. Under the assumptions of Theorem 2.8, we have

$$
\Gamma_{D_{p, q}}(x) \supset \Gamma_{D_{p^{\prime}, q^{\prime}}}(x) .
$$

Theorem 2.9. Let $p=p(n)$ be an arbitrary sequence, $q(n) \leq n$ for all $n \in \mathbb{N}$ and

$$
\lim _{n \rightarrow \infty} \frac{n}{q(n)-p(n)}=d \neq 0
$$

hold. Then, $\delta_{D_{p, q}}(K) \neq 0$ implies $\delta(K) \neq 0$ for every $K \subseteq \mathbb{N}$.

Proof. If $\delta_{D_{p, q}}(K) \neq 0$ then,

$\delta_{D_{p, q}}^{*}(K)=\limsup _{n \rightarrow \infty} \frac{|\{p(n)+1 \leq k \leq q(n): k \in K\}|}{q(n)-p(n)}>0$,

and the relation

$$
\begin{aligned}
& \frac{|\{p(n)+1 \leq k \leq q(n): k \in K\}|}{q(n)-p(n)} \leq \\
& \frac{n}{q(n)-p(n)} \cdot \frac{|\{k \leq n: k \in K\}|}{n}
\end{aligned}
$$

hold. Therefore

$$
\delta^{*}(K)=\limsup _{n \rightarrow \infty} \frac{|\{k \leq n: k \in K\}|}{n}>0
$$

Hence $\delta(K) \neq 0$. End of proof.

Let us note that if $q(n)=n$ for all $n \in \mathbb{N}$, the condition (7) is ommitted in the Theorem 2.9.

Corollary 2.4. Under the conditions of Theorem 2.9 the inclusion

$$
\Gamma(x) \supset \Gamma_{D_{p, q}}(x)
$$

hold.

\subsection{Some inclusion results for $\Gamma_{D_{\lambda}}(x)$}

In this section we consider the case $q(n):=\lambda(n)$ and $p(n):=\lambda(n-1)$ when the sequence $\lambda=\{\lambda(n)\}_{n \in \mathbb{N}}$ is a strictly increasing sequence of positive natural numbers and $\lambda(0)=0$.

Theorem 2.10. If the limit $\lim _{n \rightarrow \infty} \frac{\lambda(n)}{\lambda(n)-\lambda(n-1)}=d \neq 0$ is hold. Then, $\delta_{D_{\lambda}}(K) \neq 0$ implies $\delta_{C S_{\lambda}}(K) \neq 0$ for every subset $K$ of $\mathbb{N}$.

Proof. If $\delta_{D_{\lambda}}(K) \neq 0$ then we have

$\delta_{D_{\lambda}}^{*}(K)=\limsup _{n \rightarrow \infty} \frac{|\{k: \lambda(n-1)+1 \leq k \leq \lambda(n), k \in K\}|}{\lambda(n)-\lambda(n-1)}>0$,

and the following inequality

$$
\begin{gathered}
\frac{1}{\lambda(n)-\lambda(n-1)}|\{k: \lambda(n-1)+1 \leq k \leq \lambda(n), k \in K\}| \leq \\
\frac{\lambda(n)}{\lambda(n)-\lambda(n-1)} \frac{1}{\lambda(n)}|\{k: 1 \leq k \leq \lambda(n), k \in K\}|
\end{gathered}
$$

hold. Therefore, under the assumption we have

$\delta_{C S_{\lambda}}^{*}(K):=\limsup _{n \rightarrow \infty} \frac{1}{\lambda(n)}|\{k: 1 \leq k \leq \lambda(n), k \in K\}|>0$.

So, $\delta_{C S_{\lambda}}(K) \neq 0$. 
Corollary 2.5. Under the condition of Theorem 2.10, the inclusion

$$
\Gamma_{C S_{\lambda}}(x) \supset \Gamma_{D_{\lambda}}(x)
$$

hold.

Theorem 2.11. Let $G=\{\lambda(n)\}_{n \in \mathbb{N}}$ and $G^{\prime}=$ $\left\{\lambda^{\prime}(n)\right\}_{n \in \mathbb{N}}$ be an infinite subset of positive natural numbers. If $G^{\prime}-G$ is finite set and the limit

$$
\lim _{n \rightarrow \infty} \frac{\lambda^{\prime}(n)-\lambda^{\prime}(n-1)}{\lambda(n)-\lambda(n-1)}=d \neq 0,
$$

hold. Then, $\delta_{D_{\lambda}}(K) \neq 0$ implies $\delta_{D_{\lambda^{\prime}}}(K) \neq 0$ for every $K \subseteq \mathbb{N}$.

Proof. If the set $G^{\prime}-G$ is finite, then there exists a positive natural number $N$ such that the inclusion

$$
\left\{\lambda^{\prime}(n): n \geq N\right\} \subset G
$$

hold. It means that there exists a monotone increasing sequence $(j(n))_{n \in \mathbb{N}}$ tending infinity such that $\lambda^{\prime}(n)=$ $\lambda(j(n))$.

If $\delta_{D_{\lambda}}(K) \neq 0$, then we have

$$
\begin{aligned}
& \delta_{D_{\lambda}}^{*}(K)= \\
& \quad=\limsup _{n \rightarrow \infty} \frac{|\{k: \lambda(n-1)+1 \leq k \leq \lambda(n), k \in K\}|}{\lambda(n)-\lambda(n-1)}>0 .
\end{aligned}
$$

Also, the inequality

$$
\begin{aligned}
& \frac{|\{k: \lambda(n-1)+1 \leq k \leq \lambda(n), k \in K\}|}{\lambda(n)-\lambda(n-1)} \leq \\
& \leq \frac{\lambda(j(n))-\lambda(j(n)-1)}{\lambda(n)-\lambda(n-1)} \times \\
& \times \frac{|\{k: \lambda(j(n)-1)+1 \leq k \leq \lambda(j(n)), k \in K\}|}{\lambda(j(n))-\lambda(j(n)-1)}
\end{aligned}
$$

hold. After taking upper limit we have

$\limsup _{n \rightarrow \infty} \frac{|\{k: \lambda(j(n)-1)+1 \leq k \leq \lambda(j(n)), k \in K\}|}{\lambda(j(n))-\lambda(j(n)-1)}>0$.

Therefore,

$$
\limsup _{n \rightarrow \infty} \frac{\left|\left\{k: \lambda^{\prime}(n-1)+1 \leq k \leq \lambda^{\prime}(n), k \in K\right\}\right|}{\lambda^{\prime}(n)-\lambda^{\prime}(n-1)}>0
$$

and this gives $\delta_{D_{\lambda^{\prime}}}(K) \neq 0$. This finishes the proof.

Corollary 2.6. Let us assume that $\lim _{n \rightarrow \infty} \frac{\lambda^{\prime}(n)-\lambda^{\prime}(n-1)}{\lambda(n)-\lambda(n-1)}=$ $d \neq 0$. Then, the following statements are true:

i) If $G^{\prime}-G$ is finite, then

$$
\Gamma_{D_{\lambda}}(x) \subset \Gamma_{D_{\lambda^{\prime}}}(x)
$$

holds,

ii) If $G^{\prime} \Delta G$ is finite, then

$$
\Gamma_{D_{\lambda}}(x)=\Gamma_{D_{\lambda^{\prime}}}(x)
$$

holds.

\section{REFERENCES}

[1] R.P. Agnew, On deferred Cesaro Mean, Ann. of Math., 33 (1932), 413-421.

[2] R.P. Buck, Generalized asymptotic density, Amer. J. Math. Comm., 75 (1953), 335-346.

[3] J.S. Connor, The statistical and strong p-Cesaro convergence of sequences, Analysis, 8 (1988) 47-63.

[4] J.S. ConNOR, On strong matrix summability with respect to a modulus and statistical convergence, Canad. Math. Bull., 32 (1989), 194-198.

[5] K. Demirci, A-statistical core of a sequence, Math., 33 No2 (2000),343-353.

[6] K. Demirci, On A-statistical cluster points, Glasnik Math., Vol37 57 (2002),293-301.

[7] P. Erdös, G. Tenenbaum, Sur les densites de certaines suites d'entires, Proceeding of the London Math. Socaity, vol 59, 3 (1989), 417-438.

[8] H. FAst, Sur la convergence statistique, Colloq. Math., 2 (1951),241-244.

[9] A.R. Freedman, J.J. Sember, P. Aphael, Some Cesaro type summability Spaces, Proc. London Math. Soc., 37 (1978), 301-313.

[10] J.A. FrIDY, On statistical convergence, Analysis 5 (1985),301-313.

[11] J.A. FRIDY, Statistical limit points, Proc. of the American Math. Soc. Vol118, number4, August1993, 11871191.

[12] J.A. Fridy, C. Orhan, Statistical limit superior and statistical limit inferior, Proc. of the American Math. Soc. Vol125, number12, December1997, 3625-3631.

[13] P. Kostyrko, M. Macaj, T. Salat, O. Strouch, On statistical limit points, Proc. Amer. Math. Soc. vol129, number9,(2000),2647-2654.

[14] M. Kucukaslan, M. Yilmazturk, Deferred statistical convergence, Kyunpook Mathematical Journal, Submitted, (2013).

[15] M. Kucukaslan, M. Yilmazturk, On strongly Cesaro Summability and deferred statistical convergence of sequences, BEÜ Journal of Science and Technology, $3,(2013), 22-25$.

[16] M. Mamedov, S. Pelivan, Statistical cluster points and Turnpike Theorem in Nonconvex Problems, Journal of Mathematical Analysis and Applications 256,(2001),686-693.

[17] M. Mursaleen, $\lambda$-statistical convergence, Mathematica Slovaca, vol 50.1 (2000), 111-115.

[18] J.A. OsIKIEwICZ, Summability of matrix subnothods and Spliced Sequences, Ph.D. Thesis, Kent State University, 1997.

[19] S. Pehlivan, M. Gürdal, B. Fisher, Lacunary statistical cluster points of sequences, Mathematical communications, 11 (2006), 39-46.

[20] S. Pehlivan, H. Albayrak, H.Z. Toyganözü, The Theory of Convergence and the set of statistical Cluster Points, Advances in Dynamic System and Applications, vol6, number1, (2011),111-119. 
[21] T. SAlat, On statistically convergent sequences of real numbers, Math. Slovaca 30(1980), 139-150.

[22] I.J. Schoenberg, The integrability of certain functions and related summability methods, Amer. Math. Monthly, 66, (1959), 361-375.

[23] H. Steinhaus, Sur la convergence ordinaire et la convergence asymptotique, Colloq. Math., 2 (1951), 73-74.
[24] J. Zeager, Statistical Limit Point Theorems, Internat. J. Math. and Math. Sci., vol23, No11,(2000),741-752.

[25] A. Zygmund, Trigonometric Series, Cambiridge Univ. Press U.K. (1979). 